\title{
Enhancement of the Performance of GaP Solar Cells by Embedded In(N)P Quantum Dots
}

Yanjin Kuang ${ }^{1, *}, \mathrm{Ke} \mathrm{Sun}^{2,3}$, Supanee Sukrittanon ${ }^{4}, \mathrm{Ko} \mathrm{Takabayashi}^{5}$, Itaru Kamiya ${ }^{5}$, Nathan S. Lewis ${ }^{2,3,6,7}$ and Charles W. Tu ${ }^{8}$

1. Department of Physics, University of California, San Diego, La Jolla, CA 92093, USA.

2. Division of Chemistry and Chemical Engineering, California Institute of Technology, Pasadena, CA 91125, USA.

3. Joint Center for Artificial Photosynthesis, California Institute of Technology, Pasadena, CA 91125, USA.

4. Material Science and Engineering Program, University of California, San Diego, La Jolla, CA 92093, USA

5. Quantum Interface Laboratory, Toyota Technological Institute, Nagoya 468-8511, Japan.

6. Beckman Institute and Molecular Materials Research Center, California Institute of Technology, Pasadena, CA 91125, USA.

7. Kavli Nanoscience Institute, California Institute of Technology, Pasadena, CA 91125, USA.

8. Department of Electrical and Computer Engineering, University of California, San Diego, La Jolla, CA 92093, USA.

* Corresponding email: yakuang@ucsd.edu 


\begin{abstract}
Improving the utilization of solar spectra of wide bandgap semiconductors that can potentially provide enough free energy is one of the promising strategies for realizing efficient and spontaneous integrated conversion of solar energy to chemical fuels. We demonstrate herein nitrogen doped InP quantum dots (QDs) embedded in wide bandgap GaP could improve the solar energy conversion performance. Photoelectrochemical experiments in contact with a nonaqueous, reversible redox couple indicated that the QDembedded devices exhibited improved performance relative to devices without QDs, with short-circuit current densities increasing from $0.16 \mathrm{~mA} \mathrm{~cm}^{-2}$ for GaP-only devices to 0.23 and $0.29 \mathrm{~mA} \mathrm{~cm}^{-2}$ for InP and InNP QD-embedded devices, respectively. Additionally, the open-circuit voltages increased from $0.95 \mathrm{~V}$ for GaP-only devices to 1.11 and $1.14 \mathrm{~V}$ for InP and InNP QD-embedded devices, respectively, and the external quantum yield of the devices was also enhanced by the embedded QDs. The improvement is attributed to the absorption of sub-bandgap photons by the In(N)P QDs.
\end{abstract}

\title{
Graphical Abstract
}
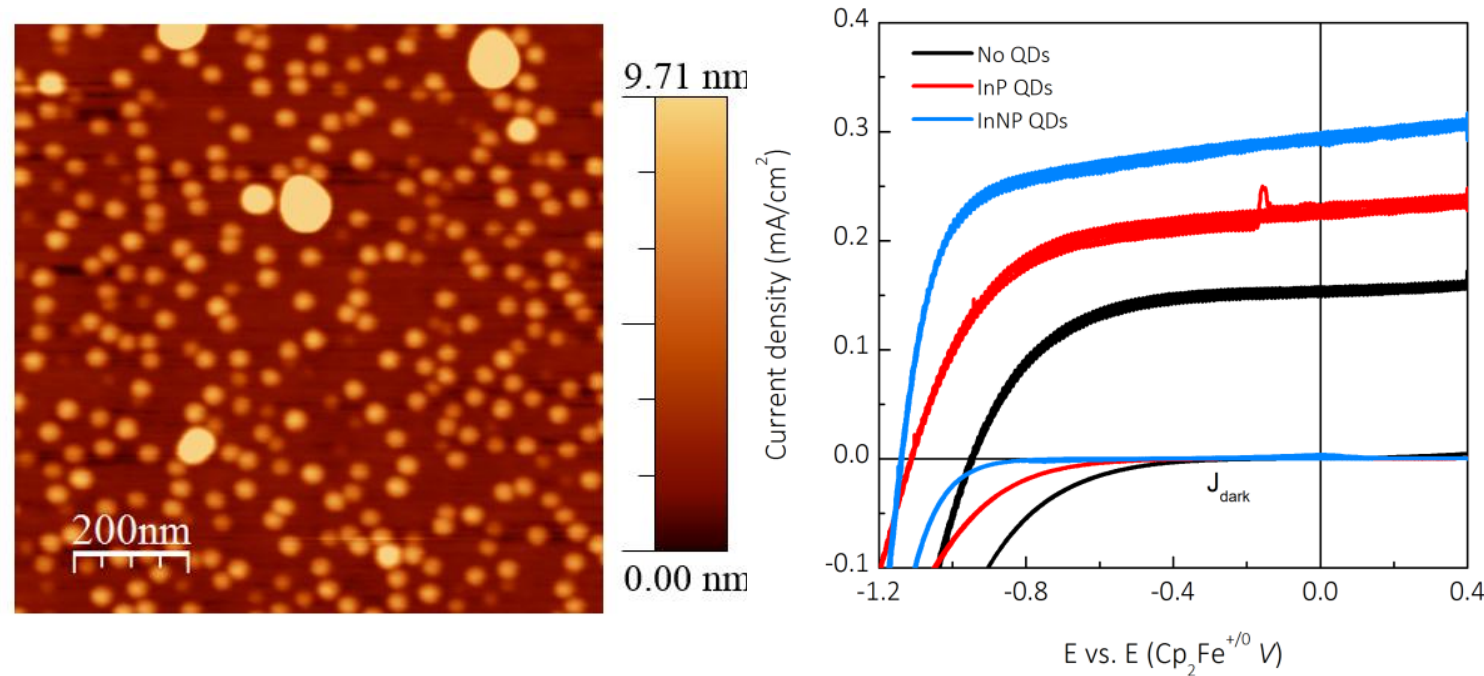

\section{Keywords}

quantum dots, InNP, solar cells, indirect bandgap engineering, MBE 


\section{Introduction}

$\mathrm{GaP}$ is not commonly used as the base absorber in terrestrial photovoltaic devices due to a bandgap $\left(\mathbf{E}_{\mathrm{g}}=2.26 \mathrm{eV}\right)$ that is too large to absorb much of the solar spectrum, and because the indirect gap requires use of a relatively thick layer of $\mathrm{GaP}(>5 \mu \mathrm{m})$ for effective absorption of light, relative to Group III-V semiconductors with direct bandgap. Nevertheless, the large bandgap and the electron affinity level $(3.8 \mathrm{eV})$ of $\mathrm{GaP}$ may prove useful in an integrated solar-fuels device. Although the bandgap of GaP is considerably higher than optimal for an efficient solar-driven water-splitting device, ${ }^{1}$ a tandem cell comprised of an $\mathrm{n}$-GaP photoanode and a photocathode such as $\mathrm{p}-\mathrm{Si}^{2}, \mathrm{p}-\mathrm{WSe}_{2}{ }^{3}$, or $\mathrm{p}$ $\mathrm{Cu}_{2} \mathrm{O}^{4,5}$ may be useful for a solar-fuels device that couples the reduction of $\mathrm{CO}_{2}$ to a fuel with the oxidation of water to $\mathrm{O}_{2}(\mathrm{~g})$, because such multi-electron chemical reduction processes may involve larger catalytic overpotentials, and thus require higher overall photovoltages, than water-splitting devices. In addition, mass-transport limitations related to the limited concentration of $\mathrm{CO}_{2}$ in the atmosphere may constrain the current density attainable for a $\mathrm{CO}_{2}$-reducing device to values compatible with those obtainable from light absorbers with larger bandgaps than are optimal for solar-driven water splitting. Furthermore, GaP has a lattice constant very similar to that of $\mathrm{Si}(5.451 \AA$ and $5.431 \AA$, respectively $)^{6}$, allowing the growth on $\mathrm{Si}$ of high-quality $\mathrm{GaP}$ without anti-phase domains, through a buffer layer < $200 \mathrm{~nm}$ thick. Optoelectronic devices have in fact been fabricated using such a platform. ${ }^{6-9}$ Thus, a monolithic tandem cell comprised of a catalyst-loaded Si photocathode and an $\mathrm{n}-\mathrm{GaP}$ photoanode, protected against corrosion and loaded with an oxygen-evolution catalyst, ${ }^{10}$ could provide an implementation of a functioning solar-fuels device. Techniques that enhance the absorption of $\mathrm{GaP}$ while reducing the thickness required for effective absorption of light, such as bandgap engineering $^{11,12}$ and nanostructuring ${ }^{13}$, will increase the utility of GaP not only for photoelectrochemical applications, but also for photovoltaic and optoelectronic applications.

One technique for tailoring the optoelectronic properties of GaP-based devices is embedment of quantum dots (QDs) into the GaP matrix. Self-assembled InP QDs can be fabricated on GaP substrates via the coherent-island Stranski-Krastanov growth mode ${ }^{14}$, 
and a light-emitting diode (LED) with an emission energy of $\sim 2 \mathrm{eV}$ has been fabricated using this materials system ${ }^{15}$. The InP/GaP QD system has a band alignment intermediate between types $\mathrm{I}$ and $\mathrm{II}^{14}$, allowing further tailoring of the optoelectronic properties of the assembly through the incorporation of $\mathrm{N}$ into the QDs, to form dilute-nitride InNP QDs ${ }^{16}$. Herein we report the performance of a p-i-n GaP device that contains embedded InP and InNP QDs. The QDs were fabricated on $\mathrm{n}-\mathrm{GaP}(100)$ substrates $\left(\mathrm{n} \sim 1 \times 10^{18} \mathrm{~cm}^{-3}\right)$ via the self-assembly process using gas-source molecular-beam epitaxy (GSMBE). Embedment of InP and InNP QDs into GaP p-i-n cells increased the absorption and enhanced both the short-circuit current density $\left(J_{\mathrm{sc}}\right)$ and the open-circuit voltage $\left(V_{\mathrm{oc}}\right)$ of the resulting devices, relative to structures that did not have embedded QD's in the material.

\section{Experimental}

\section{Gas-Source Molecular Beam Epitaxy}

A Varian Gen-II MBE system, modified to thermally crack gas-phase $\mathrm{PH}_{3}$ to $\mathrm{P}_{2}$ and $\mathrm{H}_{2}$, and $\mathrm{GaP}(001)$ substrates, were used to fabricate all samples described in this work. Elemental $\mathrm{Ga}$ and $\mathrm{In}$, solid $\mathrm{Si}$, solid $\mathrm{Be}$, and $\mathrm{N}$ radicals produced using radio-frequency plasma activation were used as Group III sources, n-type dopant, p-type dopant and N source, respectively. The native oxide was removed from semi-insulating GaP substrates using a $\mathrm{P}_{2}$ overpressure at $\sim 580{ }^{\circ} \mathrm{C}$. For single-layer $\mathrm{In}(\mathrm{N}) \mathrm{P}$ QD samples prepared for optical-quality studies, a $200 \mathrm{~nm}$ thick GaP buffer layer was grown at a rate of $1 \mu \mathrm{m} \mathrm{h}^{-1}$ after desorption of the oxide layer. The growth was then interrupted and the substrate temperature was lowered to $440-480{ }^{\circ} \mathrm{C}$. The temperature of the In Knudsen cell was raised until a deposition rate of 0.1 monolayer $\mathrm{s}^{-1}\left(\mathrm{ML} \mathrm{s}^{-1}\right)$, as calibrated by reflection high-energy electron-diffraction (RHEED) intensity oscillation, was reached ${ }^{17}$. For InNP QDs, the $\mathrm{N}$ plasma was activated and set to a plasma power of $280 \mathrm{~W}$ using a $\mathrm{N}_{2}$ flow rate of $1.2 \mathrm{sccm}$ before the InNP QD formation process. The InP (InNP) QD selfassembly process began when the In (In and N) shutters were opened. When the InP (InNP) wetting layer exceeded the critical thickness, the InP (InNP) QDs self-assembled under a large compressive strain. The formation of QDs was confirmed by a change in the in-situ RHEED pattern from a streaky pattern to a chevron pattern, and was also 
confirmed by atomic-force microscopy (AFM) images of the uncapped QD samples. Two ML of InP (InNP) were deposited in total for all samples in this study. After deposition of the QDs, a $20 \mathrm{~nm} \mathrm{GaP}$ cap layer was grown at the temperature used for formation of the QDs to maintain the QD morphology and prevent indium desorption (due to its higher vapor pressure relative to gallium) during subsequent temperature increase. Note that all of the QDs in this study are smaller than $7 \mathrm{~nm}$ in height (figure 2) and no QDs should be exposed beyond the $20 \mathrm{~nm} \mathrm{GaP}$ cap layer, which is confirmed in situ by the recovery of the streaky RHEED pattern during growth. An additional GaP cap layer, $30 \mathrm{~nm}$ in thickness, was grown at $580{ }^{\circ} \mathrm{C}$ on samples prepared for photoluminescence (PL) studies. If no cap layer were grown on top of the QDs, the QDs would oxidize once they are taken out of the vacuum chamber. This oxidation process degrades the quality of the QDs and significantly reduces the PL intensity. The photo-excited carriers would also tend to recombine through the surface states (mostly from the dangling bonds) on the QDs through the Shockley-Read-Hall mechanism. This non-radiative recombination would quench the PL signal intensity. Moreover, the uncapped QDs see a high surface potential (atmosphere) which can result in a shift of the quantum confined energy level and hence a shift of the PL peak position ${ }^{18}$. Therefore, a $50 \mathrm{~nm}$-thick capping layer was used throughout the optical and device study to prevent device oxidation and degradation and to maintain the optical response consistency. For the In(N)P QD-embedded GaP solarcell devices, a $200 \mathrm{~nm}$ thick GaP layer with a dopant concentration of $n=1 \times 10^{18} \mathrm{~cm}^{-3}$ and an undoped $50 \mathrm{~nm}$ thick GaP layer were grown after desorption of the native oxide from the $\mathrm{GaP}(100)$ substrates $\left(n \sim 1 \times 10^{18} \mathrm{~cm}^{-3}\right)$ at $\sim 580{ }^{\circ} \mathrm{C}$, but before the growth of eight periods of $\operatorname{In}(\mathrm{N}) \mathrm{P}$ QDs. Each period of the QD structure consisted of a $2 \mathrm{~nm}$ thick deltadoped layer $\sim 1 \times 10^{11} \mathrm{~cm}^{-2}$, an In(N)P QD layer grown at $460{ }^{\circ} \mathrm{C}$, a $20 \mathrm{~nm}$ thick GaP cap layer grown at $460{ }^{\circ} \mathrm{C}$, and a $30 \mathrm{~nm}$ thick GaP cap layer grown at $580{ }^{\circ} \mathrm{C}$ (to smooth the surface for the subsequent period of QD growth). An undoped $50 \mathrm{~nm}$ thick GaP spacer layer was grown after the final period of QD growth, followed by a $100 \mathrm{~nm}$ thick $\mathrm{p}-\mathrm{GaP}$ layer $\left(p=1 \times 10^{18} \mathrm{~cm}^{-3}\right)$, and a $\mathrm{p}^{+}-\mathrm{GaP}$ contact layer $\left(\mathrm{p}=3 \times 10^{18} \mathrm{~cm}^{-3}\right)$ that was $50 \mathrm{~nm}$ in thickness. An identical GaP p-i-n structure without any embedded QDs was also grown for comparison purposes. Previous studies showed that rapid thermal annealing (RTA) improves the PL intensity by improving the optical quality of samples, ${ }^{17}$ so the capped 
QD samples and QD devices used in this study were covered by a GaP wafer (to protect the surface) and were annealed at $800{ }^{\circ} \mathrm{C}$ for $30 \mathrm{~s}$ prior to characterization.

\section{Atomic Force Microscopy and Photoluminescence Measurements}

Ex situ atomic-force microscopy (AFM) studies were performed under a vacuum pressure of $\sim 10^{-3} \mathrm{~Pa}$ using the dynamic force microscopy mode (SII Nanotechnology, ESWEEP) with a Si cantilever.

For photoluminescence (PL) measurements, the samples were cooled to $4 \mathrm{~K}$ in an evacuated cryostat. A continuous-wave $532 \mathrm{~nm}$ diode laser was used as an excitation source and the power density was set to $\sim 1 \mathrm{~W} \mathrm{~cm}^{-2}$. The PL was dispersed by a monochromator (Princeton Instruments, SpectraPro 2300i) and detected by a cooled SiCCD (Princeton Instruments, PIXIS 256).

\section{Fabrication of Electrodes and Electrochemistry}

The contact fabrication, chemical purification, and measurement methods have been described previously ${ }^{19}$. The substrate with In as ohmic contact was attached to a coiled, tin-plated $\mathrm{Cu}$ wire using conductive Ag paint, and epoxy (Hysol 9460F) was used to seal, face-down, the assembly at the end of a segment of glass tubing. The active area of each electrode was determined by the epoxy and measured using an optical scanner, with a typical value of $0.1-0.2 \mathrm{~cm}^{2}$. Electrodes were left at room temperature to dry overnight. Before loading into the glovebox for photoelectrochemical measurement, the photoelectrodes were etched in a solution of $0.04 \%(\mathrm{v} / \mathrm{v}) \mathrm{Br} 2$ in methanol $(\mathrm{CH} 3 \mathrm{OH}$, low water, J. T. Baker) for $15 \mathrm{~s}$, followed by immersion in $1.0 \mathrm{M} \mathrm{KOH}(\mathrm{aq})$ for $30 \mathrm{~s}$. The electrode was thoroughly rinsed with water and $\mathrm{CH} 3 \mathrm{OH}$, then dried in a flow of $\mathrm{N} 2(\mathrm{~g})$, and quickly transferred into a glovebox that was continually purged with ultra-high purity (UHP) Ar. Electrochemical data were obtained using a Biologic SP200 potentiostat. The nonaqueous electrochemical solutions each contained $1.0 \mathrm{M} \mathrm{LiClO}_{4}, 0.5 \mathrm{mM}$ ferrocenium, and $90 \mathrm{mM}$ ferrocene. The electrolyte solution was rapidly stirred with a small, Teflon-covered stir bar. Illumination was provided with an ELH-type tungstenhalogen lamp. The light intensity was determined by the use of a Si photodiode (Thorlab) that was previously calibrated relative to a secondary standard photodetector that was NIST-traceable and calibrated at $100 \mathrm{~mW} \mathrm{~cm}^{-2}$ of Air Mass (AM) 1.5 illumination. 
Electrodes were washed with $\mathrm{CH} 3 \mathrm{CN}$ prior to transfer to the electrolyte solutions. The working photoelectrodes were oriented vertically facing downward toward the incident light, with the light intensity adjusted by use of neutral density filters. To obtain the current density vs. potential (J-E) data, the potential was swept at a rate of $40 \mathrm{mV} \mathrm{s}^{-1}$ in a three-electrode setup using a Pt mesh and a Pt wire as the counter electrode and reference electrode, respectively. External quantum yield measurements used the same electrochemical setup as that used for the collection of the J-E data. The J-E data were corrected for the solution resistance loss calculated from extrapolation of the linear portion of the J-E data measured using Pt disc electrode as a working electrode. The uncompensated resistance was measured to be $0.0057 \mathrm{ohm}-\mathrm{cm}^{2}$.

\section{Results and Discussion}

Figure 1 presents a schematic illustration of the structures of the GaP and QD-embedded $\mathrm{GaP}$ devices that were fabricated for photoelectrochemical studies. Eight periods of $\mathrm{In}(\mathrm{N}) \mathrm{P}$ QDs were grown under high compressive strain within the undoped GaP layer of a GaP-based p-i-n structure. Optical-quality samples that did not contain either the p-GaP buffer $\left(\mathrm{p}=1 \times 10^{18} \mathrm{~cm}^{-3}\right)$ layer or the $\mathrm{p}^{+}-\mathrm{GaP}$ contact layer, and that contained only a single layer of In(N)P QDs, were used for photoluminescence studies. The formation of QDs was confirmed by a change in the in-situ reflection high-energy electron-diffraction (RHEED) pattern from a streaky pattern to a chevron pattern, and by atomic-force microscopy (AFM) images of the uncapped QD samples (Figure 2).

The AFM images were also used to calculate the densities and heights of the InP and InNP QDs. The densities of the InP and InNP QDs were $174 \pm 20 \mu \mathrm{m}^{-2}$ and $230 \pm 34 \mu \mathrm{m}^{-2}$, respectively. The heights of the InP and InNP QDs were 4.5 $\pm 1.1 \mathrm{~nm}$ and $5.8 \pm 1.3 \mathrm{~nm}$, respectively. The presence of a $\mathrm{N}$ plasma during the QD assembly process did not affect the morphology significantly, although aggregated clusters with diameters of $\sim 100 \mathrm{~nm}$ and heights of $\sim 30 \mathrm{~nm}$ were found more frequently on InNP samples than on InP samples, possibly due to an increased rate of nucleation when the reactive $\mathrm{N}$ plasma was present. 
Although such clusters are too large to contribute to the confinement energy in the PL spectra, the PL was significantly modified by the addition of $\mathrm{N}$ into the QDs. Figure 3 shows the PL spectra at $4 \mathrm{~K}$ for GaP samples embedded with InP QDs or InNP QDs, and the inset to Figure 3 shows a schematic band diagram for the $\operatorname{In}(\mathrm{N}) \mathrm{P} / \mathrm{GaP}$ QD system based on a published framework. ${ }^{20}$ The PL was done in $4 \mathrm{~K}$ to increase luminescence intensity and better resolve the peak positions from InP QDs and InNP QDs. The PL intensity of InNP QD-embedded samples was significantly higher than that of InP QDembedded samples, in contrast to the $\mathrm{GaIn}(\mathrm{N}) \mathrm{As} / \mathrm{GaAs}$ system wherein PL intensity decreases are associated with $\mathrm{N}$ incorporation due to $\mathrm{N}$-related defects. ${ }^{21}$ Compressively strained InP QDs having a size comparable to the QDs embedded in the samples described herein are expected to have $\Gamma$-like quantum-confined conduction-band ground states lying at higher energies than the X-band energy of the GaP matrix, causing electrons to occupy states at the InP/GaP interface. ${ }^{20}$ Therefore, electrons tend to recombine with heavy holes inside the InP QDs in a spatially indirect way (dashed arrow, Figure 3 inset). However, localized states are formed during the incorporation of $\mathrm{N}$ into InNP QDs, ${ }^{22}$ and the localized states hinder the thermalization of electrons into X-derived interface states prior to recombination. Electrons trapped at the $\Gamma$-like quantum-confined ground states ( $E_{-}^{I n N P}$, solid green line, Figure 3 inset) and the N-related states lying directly below (the exponential dilute-nitride band-tail states, ${ }^{23}$ green gradient, Figure 3 inset) can recombine with the heavy holes within the QDs in a spatially direct way ${ }^{24}$ (solid red arrow, Figure 3 inset), resulting in a blue-shifted PL peak and an increase in the recombination efficiency ${ }^{25}$. The GaP matrix itself has an indirect bandgap and accordingly did not exhibit any appreciable PL.

The introduction of $\mathrm{N}$ into the QDs also significantly affected the photoelectrochemical performance of QD-embedded p-i-n GaP devices. Electrochemical measurements were performed using a three-electrode configuration using working electrodes constructed from the p-i-n GaP and $\operatorname{In}(\mathrm{N}) \mathrm{P}$ QD-embedded p-i-n GaP devices (such that the $\mathrm{p}^{+}-\mathrm{GaP}$ layer was in contact with the electrolyte solution), a Pt mesh as the counter electrode, and a Pt wire poised at the solution potential as the reference electrode. The electrodes were illuminated by an ELH-type tungsten-halogen lamp that was calibrated to provide $100 \mathrm{~mW} \mathrm{~cm}^{-2}$ of AM 1.5 illumination at the surface of the samples. Figure 4 shows the 
current-density versus potential $(J-E)$ data for illuminated GaP and QD-embedded GaP photoelectrodes in contact with a $\mathrm{CH}_{3} \mathrm{CN}$ solution that contained the kinetically facile one-electron ferrocenium-ferrocene $\left(\mathrm{FeCp}_{2}{ }^{+/ 0}\right)$ redox couple and $\mathrm{LiClO}_{4}$ as a supporting electrolyte. The kinetically facile one-electron reversible redox couple ensured that interfacial electron-transfer processes were not rate-limiting and thus allowed measurement of the intrinsic energy-conversion properties of the photoelectrodes. All of the GaP and QD-embedded GaP electrodes produced photocurrent densities $<400 \mu \mathrm{A}$ $\mathrm{cm}^{-2}$, which is low relative to the value of $J_{\mathrm{sc}}$ obtained from other devices prepared using similar methods ${ }^{26}\left(\sim 1 \mathrm{~mA} \mathrm{~cm}^{-2}\right)$, because the electrodes were only $1 \mu \mathrm{m}$ thick, and thus did not fully absorb the incident light. The $J_{\mathrm{sc}}$ of the illuminated GaP photoelectrode was $\sim 0.16 \mathrm{~mA} \mathrm{~cm}^{-2}$, while the InP QD-embedded $\mathrm{GaP}$ photoelectrode exhibited $J_{\mathrm{sc}}$ $\sim 0.23 \mathrm{~mA} \mathrm{~cm}^{-2}$ and the InNP QD-embedded GaP photoelectrode exhibited $J_{\mathrm{sc}} \sim 0.29 \mathrm{~mA}$ $\mathrm{cm}^{-2}$. In the InAs QD-embedded GaAs system, intermediate-band absorption from embedded QDs has been shown to produce an increase in $J_{\mathrm{sc}}$ but reduces the open-circuit voltage $\left(V_{\mathrm{oc}}\right) .{ }^{27}$ In contrast, $V_{\mathrm{oc}}$ was improved for the QD-embedded GaP photoelectrodes described herein, with InNP QD-embedded photoelectrodes yielding $V_{\mathrm{oc}}=1.14 \pm 0.01 \mathrm{~V}$ and InP QD-embedded photoelectrodes yielding $V_{\text {oc }}$ of $1.11 \pm 0.01 \mathrm{~V}$, as compared to the GaP photoelectrode which yielded $V_{\mathrm{oc}}=0.95 \pm 0.01 \mathrm{~V}$. These results indicate that the embedment of the $\operatorname{In}(\mathrm{N}) \mathrm{P}$ QDs into the GaP matrix did not degrade the quality of the solid/liquid or solid-state junctions in the system.

$V_{\mathrm{oc}}$ is related to $J_{\mathrm{sc}}$ as follows:

$$
V_{o c}=\frac{n k_{B} T}{q} \ln \left(\frac{J_{s c}}{J_{o}}+1\right),
$$

where $n$ is the ideality factor, $k_{B}$ is the Boltzmann constant, $T$ is the absolute temperature, $q$ is the unsigned charge of an electron and $J_{0}$ is the exchange current density of the diode. The embedment of $\operatorname{In}(\mathrm{N}) \mathrm{P}$ QDs into the GaP matrix led to an increase in both $J_{\mathrm{sc}}$ and $V_{\mathrm{oc}}$ (Table 1). The increase in $V_{\mathrm{oc}}, 0.16 \mathrm{~V}$ and $0.19 \mathrm{~V}$ for InP and InNP QDs, respectively, however, exceeded the increase that can be attributed solely to the logarithmic contribution of the increased $J_{\mathrm{sc}}$. When $\operatorname{In}(\mathrm{N}) \mathrm{P}$ QDs were embedded in the devices, midgap traps were created in the space charge region. The QDs also induce generationrecombination processes with sub-bandgap energies, as will be discussed later. These 
make the In(N)P devices deviate substantially from the ideal diode where the ideality factor $n=1$ corresponds to radiation processes in the neutral charge region. Therefore we attribute the increase in $\mathrm{V}_{\mathrm{oc}}$ of $\operatorname{In}(\mathrm{N}) \mathrm{P}$ devices to both increase in $\mathrm{J}_{\mathrm{sc}}$ and $\mathrm{n}$.

\begin{tabular}{|c|c|c|}
\hline Sample & $J_{\mathrm{sc}}\left(\mathrm{mA} \mathrm{cm}^{-2}\right)$ & $V_{\mathrm{oc}}(\mathrm{V})$ \\
\hline No QDs & $0.16 \pm 0.01$ & $0.95 \pm 0.01$ \\
\hline InP QDs & $0.23 \pm 0.01$ & $1.11 \pm 0.01$ \\
\hline InNP QDs & $0.29 \pm 0.01$ & $1.14 \pm 0.01$ \\
\hline
\end{tabular}

Table 1. Summary of short-circuit current densities $\left(J_{\mathrm{sc}}\right)$ and open-circuit voltages $\left(V_{\mathrm{oc}}\right)$ measured for GaP (no QDs) and QD-embedded GaP devices in contact with a nonaqueous solution containing the $\mathrm{FeCp}_{2}{ }^{+/ 0}$ redox couple and $\mathrm{LiClO}_{4}$ as the supporting electrolyte.

Figure 5 shows the spectral response and external quantum yield $\left(\Phi_{\text {ext }}\right)$ measured for the devices. The InNP QD-embedded devices yielded higher $\Phi_{\text {ext }}$ values than the GaP and InP QD-embedded GaP devices. At wavelengths $<549 \mathrm{~nm}$, for which the photon energies exceeded the bandgap of GaP, the InP QD-embedded device yielded a slightly smaller $\Phi_{\text {ext }}$ relative to the GaP device, possibly indicating that the InP QD embedment affected the quality of the junction. However, the InNP QD-embedded device showed unambiguously higher $\Phi_{\text {ext }}$. This behavior can be attributed to the reduced strain and better crystal quality in InNP QDs due to the smaller $\mathrm{N}$ atom size and $\mathrm{N}$ lattice hardening effect $^{28}$. Specifically, studies have shown that the $\mathrm{N}$ induced lattice hardening effect slows down lattice mismatch related relaxation and improve optical performance of dilute nitrides. Such properties can also be attributed to the decreases in $J_{0}$ in devices with InNP QDs. The $\Phi_{\text {ext }}$ values of the QD-embedded GaP samples were significantly enhanced at wavelengths $>550 \mathrm{~nm}$ relative to the $\mathrm{GaP}$ samples, and $\Phi_{\text {ext }}$ for the InNP QD-embedded GaP sample showed a significant peak near $600 \mathrm{~nm}$, in good agreement 
with the PL data. Because the intensity of the solar spectrum is stronger for the wavelengths in the region absorbed by the $\operatorname{In}(\mathrm{N}) \mathrm{P}$ QDs $(\sim 550-650 \mathrm{~nm})$ than for wavelengths in the region absorbed by the GaP matrix $(<548 \mathrm{~nm})$, the QDs significantly increase the amount of sunlight absorbed by the devices. These results indicate that the embedded QDs resulted in enhanced absorption and in the increased $J_{\mathrm{sc}}$ value observed in the $J-E$ data. The GaP matrix itself, which was much thicker than the QD layers, absorbed most of the photons with energies higher than the bandgap of GaP, and thus the effect of QD embedment only becomes significant when the QDs absorb photons with energies below the bandgap of $\mathrm{GaP}$, which otherwise would not be absorbed by the device.

The device design also contributes to the significant improvement in performance observed for the QD-embedded GaP devices relative to $\mathrm{GaP}$ devices without embedded QDs. The $\mathrm{In}(\mathrm{N}) \mathrm{P}$ QDs were embedded within an undoped $\mathrm{GaP}$ region which is completely depleted, allowing the strong built-in electric field $\left(\sim 4000 \mathrm{~V} \mathrm{~cm}^{-1}\right)$ to quickly sweep the electron-hole pairs across the junction to the contact layers. The built-in electric field also assists thermal hopping of photogenerated carriers out of the In(N)P/GaP interface and N-related localized states.

In principle, the photocurrent density of the GaP devices could be further enhanced by increasing the loading of the QDs. In practice, however, only fabrication of 8-10 layers (depending on the size of the QDs) is feasible due to the accumulation of strain. Use of a strain-balancing technique whereby a tensile strain layer is introduced between the QD layers can increase the number of QD layers that can be fabricated. Previously, the strain-balancing technique has enabled more than 100 layers stack of InAs QDs ${ }^{29}$, considerably increasing the photoabsorption. Although dilute-nitride GaNP could be used for tensile strain layers in the $\mathrm{In}(\mathrm{N}) \mathrm{P}$ QD-embedded GaP devices, emission from GaNP, in the range $\sim 580-650 \mathrm{~nm}$ depending on the $\mathrm{N}$ composition, would prevent unambiguous quantification of the photocurrent contribution of the $\operatorname{In}(\mathrm{N}) \mathrm{P}$ QDs. Although a GaNP strain-balancing layer was not used for this demonstration, we expect that such layers would lead to improved devices. With careful control of $\mathrm{N}$ composition and hence strain manipulation, layers of high crystal quality $\operatorname{In}(\mathrm{N}) \mathrm{P}$ QDs with GaNP strain balancing layers can be fabricated with GSMBE. In addition, if $\mathrm{In}(\mathrm{N}) \mathrm{P} / \mathrm{GaP}$ were to be integrated 
onto the already demonstrated GaP/Si platform ${ }^{9}$, the materials system would be more scalable and affordable.

\section{Conclusions}

InNP QDs have much stronger PL than InP QDs due to the special bandgap alignment in these QD systems. Embedment of InP and InNP QDs, particularly InNP QDs, into GaP solar cells significantly increased the amount of light absorbed by the devices without degrading the quality of the junctions, and increases in both $J_{\text {sc }}$ and $V_{\text {oc }}$ were observed for the QD-embedded devices. Embedment of InNP QDs within a GaP matrix may increase the utility of $\mathrm{GaP}$ for photovoltaic and solar fuels generation.

\section{Acknowledgements}

Material growth by GSMBE was supported by the National Science Foundation under Grant No. DMR-0907652 and DMR-1106369. SS acknowledges the financial support from the Royal Government of Thailand Scholarship. KS and NL acknowledge the Joint Center for Artificial Photosynthesis, a DOE Energy Innovation Hub supported through the Office of Science of the U.S. Department of Energy under Award No. DE-SC0004993. KT and IK acknowledge financial supports from the Strategic Research Infrastructure Project, MEXT and Toyoaki Scholorship Foundation, Japan. 


\section{References}

1. S. Hu, C. X. Xiang, S. Haussener, A. D. Berger and N. S. Lewis, Energy \& Environmental Science 6 (10), 2984-2993 (2013).

2. S. W. Boettcher, E. L. Warren, M. C. Putnam, E. A. Santori, D. Turner-Evans, M. D. Kelzenberg, M. G. Walter, J. R. McKone, B. S. Brunschwig, H. A. Atwater and N. S. Lewis, J Am Chem Soc 133 (5), 1216-1219 (2011).

3. J. R. McKone, A. P. Pieterick, H. B. Gray and N. S. Lewis, J Am Chem Soc 135 (1), 223-231 (2012).

4. A. Paracchino, V. Laporte, K. Sivula, M. Gratzel and E. Thimsen, Nat Mater 10 (6), 456461 (2011).

5. C. X. Xiang, G. M. Kimball, R. L. Grimm, B. S. Brunschwig, H. A. Atwater and N. S. Lewis, Energy \& Environmental Science 4 (4), 1311-1318 (2011).

6. T. Katoda and M. Kishi, J Electron Mater 9 (4), 783-796 (1980).

7. T. J. Grassman, M. R. Brenner, S. Rajagopalan, R. Unocic, R. Dehoff, M. Mills, H. Fraser and S. A. Ringel, Appl Phys Lett 94 (23), 232106 (2009).

8. Y. J. Kuang, S. Sukrittanon, H. Li and C. W. Tu, Appl Phys Lett 100 (5), 053108 (2012). 
9. Y. Song and M. Larry Lee, Appl Phys Lett 103 (14), 251904 (2013).

10. S. Hu, M. R. Shaner, J. A. Beardslee, M. Lichterman, B. S. Brunschwig and N. S. Lewis, Science 344 (6187), 1005-1009 (2014).

11. J. Wu, W. Walukiewicz, K. M. Yu, J. W. Ager, E. E. Haller, Y. G. Hong, H. P. Xin and C. W. Tu, Physical Review B 65 (24), 241303 (2002).

12. A. Dobrovolsky, S. Sukrittanon, Y. Kuang, C. W. Tu, W. M. Chen and I. A. Buyanova, Small 10 (21), 4403 (2014).

13. S. Sukrittanon, Y. J. Kuang, A. Dobrovolsky, W.-M. Kang, J.-S. Jang, B.-J. Kim, W. M. Chen, I. A. Buyanova and C. W. Tu, Appl Phys Lett 105 (7), 07210 (2014).

14. F. Hatami, W. T. Masselink, L. Schrottke, J. W. Tomm, V. Talalaev, C. Kristukat and A. R. Goñi, Phys Rev B 67 (8), 085306 (2003).

15. F. Hatami, W. T. Masselink and L. Schrottke, Appl Phys Lett 78 (15), 2163-2165 (2001).

16. W. G. Bi and C. W. Tu, J Appl Phys 80 (3), 1934-1936 (1996).

17. Y.-J. Kuang, S.-W. Chen, H. Li, S. K. Sinha and C. W. Tu, Journal of Vacuum Science \& Technology B: Microelectronics and Nanometer Structures 30 (2), 02B121 (2012).

18. Y. A. Ziad, J. E. Marega, M. Mortazavi and G. J. Salamo, Nanotechnology 19 (33), 335712 (2008).

19. S. Hu, C.-Y. Chi, K. T. Fountaine, M. Yao, H. A. Atwater, P. D. Dapkus, N. S. Lewis and C. Zhou, Energy \& Environmental Science 6 (6), 1879-1890 (2013).

20. A. J. Williamson, A. Zunger and A. Canning, Phys Rev B 57 (8), R4253-R4256 (1998).

21. M. Sopanen, H. Xin and C. Tu, Appl Phys Lett 76 (8), 994-996 (2000).

22. P. R. C. Kent and A. Zunger, Phys Rev Lett 86 (12), 2613-2616 (2001).

23. I. Buyanova, W. Chen, G. Pozina, J. Bergman, B. Monemar, H. Xin and C. Tu, Appl Phys Lett 75 (4), 501-503 (1999).

24. R. Kudrawiec, G. Sek, J. Misiewicz, L. Li and J. Harmand, The European Physical Journal Applied Physics 27 (1-3), 313-316 (2004).

25. Y. J. Kuang, K. Takabayashi, S. Sukrittanon, J. L. Pan, I. Kamiya and C. W. Tu, Appl Phys Lett 105 (17) (2014).

26. C. R. Allen, J.-H. Jeon and J. M. Woodall, Sol. Energy Mater. Sol. Cells 94 (5), 865-868 (2010).

27. C. G. Bailey, D. V. Forbes, S. J. Polly, Z. S. Bittner, Y. Dai, C. Mackos, R. P. Raffaelle and S. M. Hubbard, Photovoltaics, IEEE Journal of 2 (3), 269-275 (2012).

28. J. Schöne, E. Spiecker, F. Dimroth, A. W. Bett and W. Jäger, Appl Phys Lett 92 (8), 081905 (2008).

29. J. Inoue, T. Isu, K. Akahane and M. Tsuchiya, Appl Phys Lett 89 (15), 151117 (2006). 

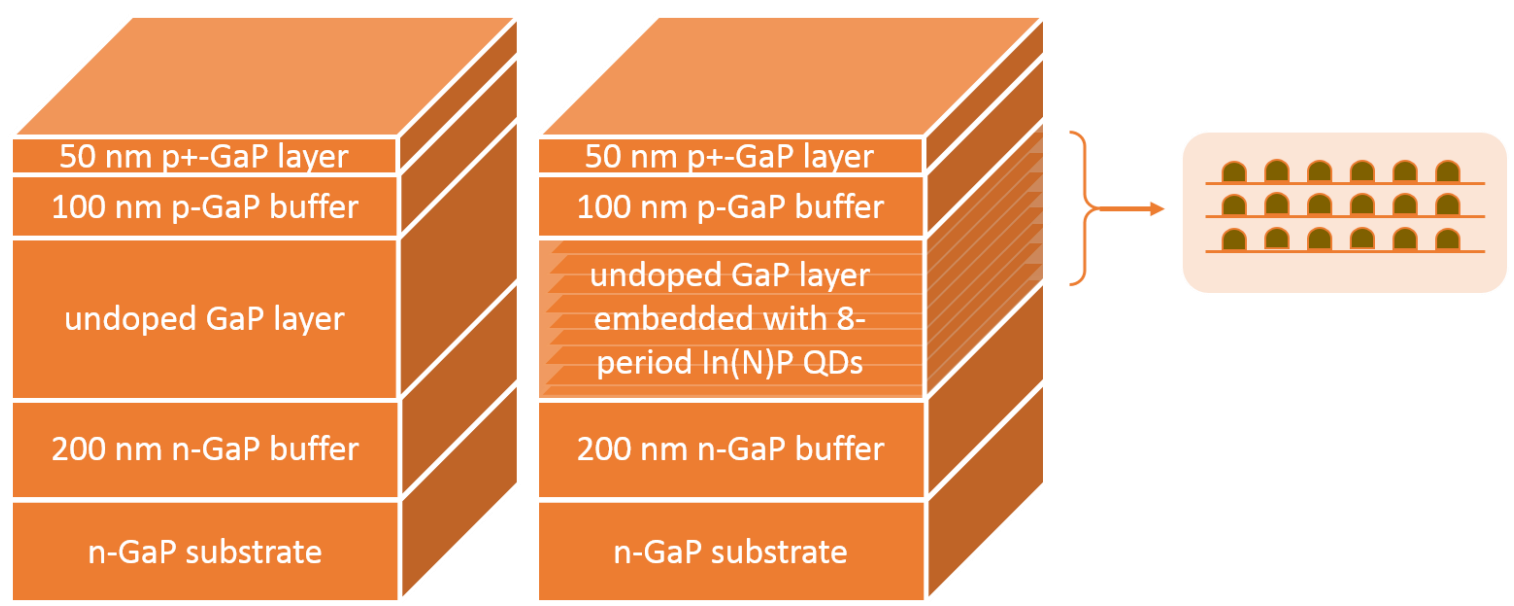

Figure 1. 

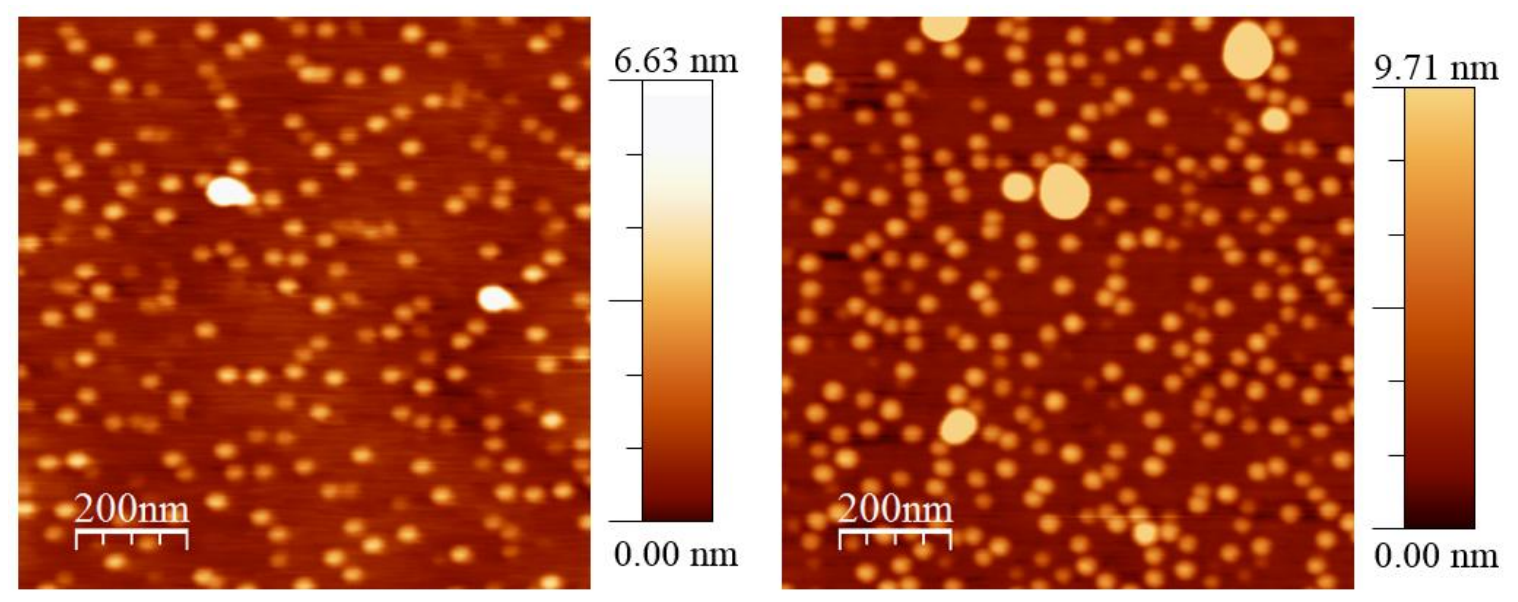

Figure 2. 


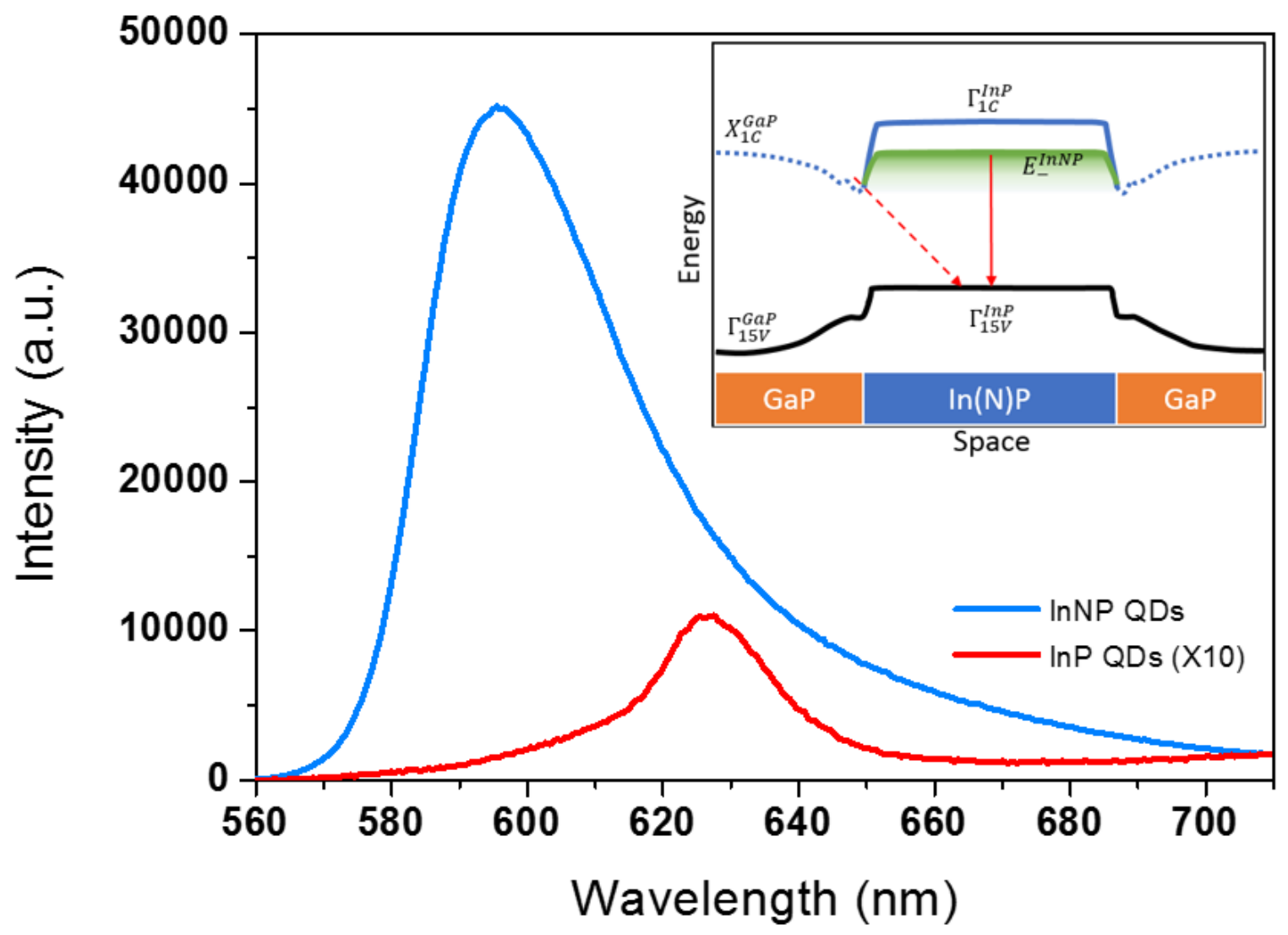

Figure 3. 


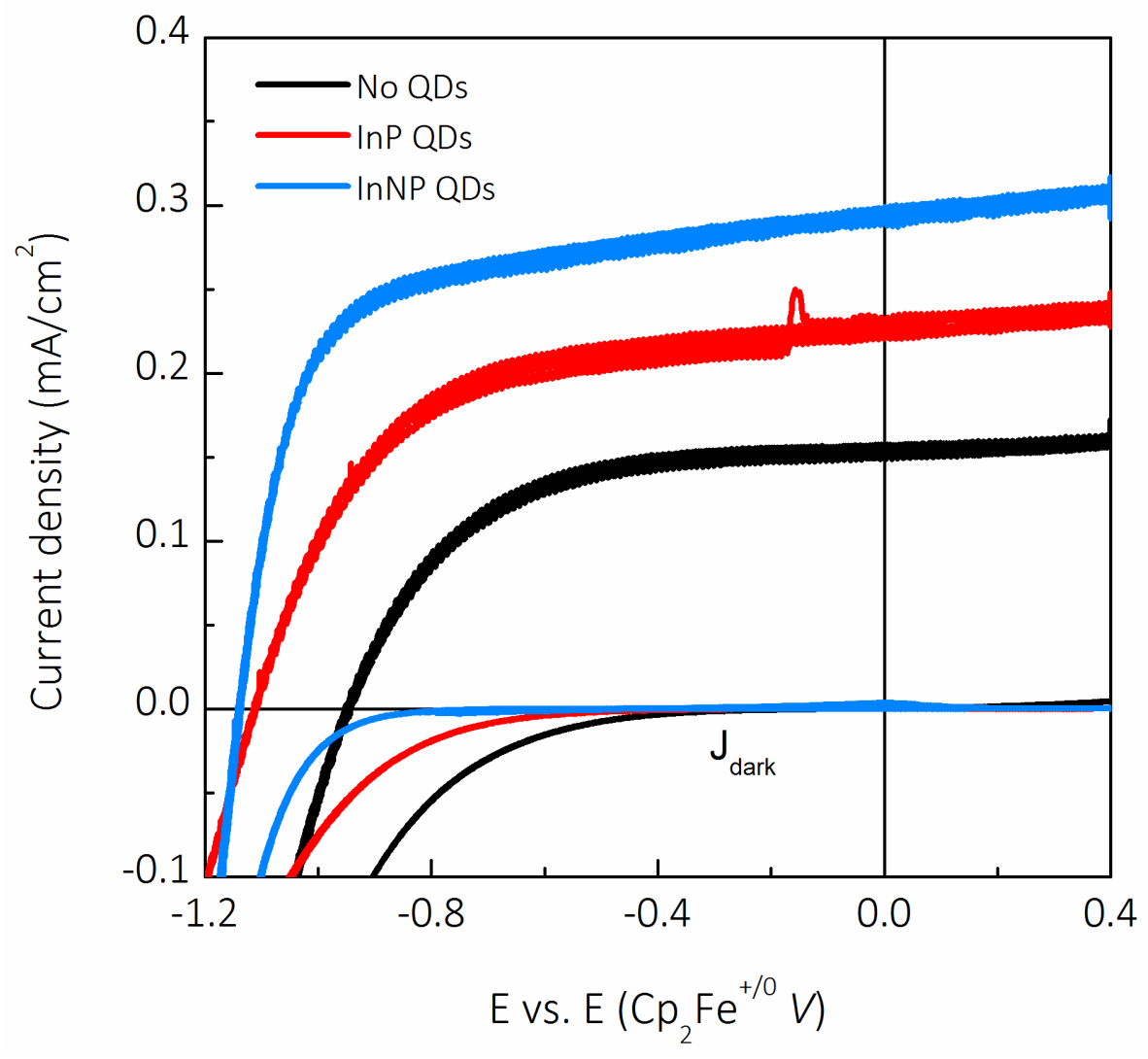

Figure 4. 


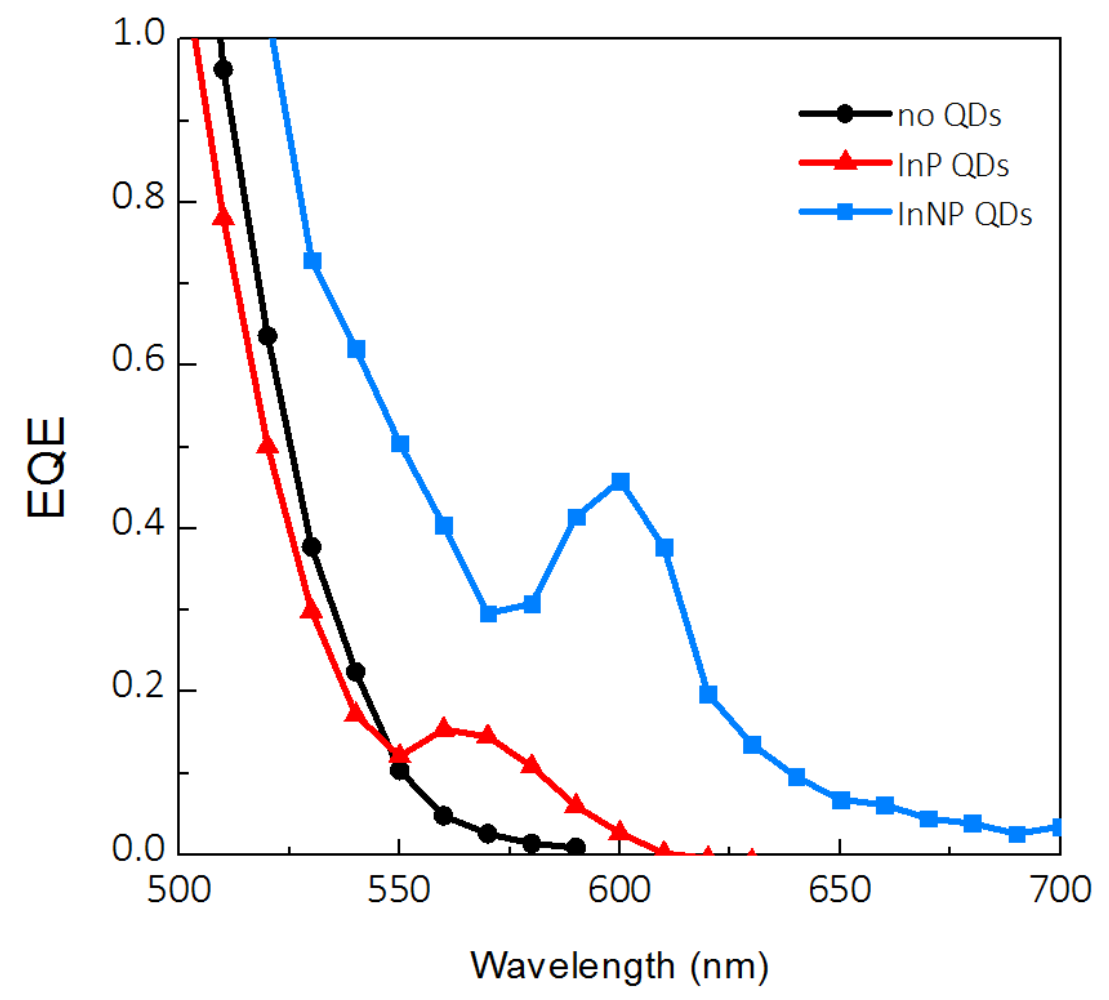

Figure 5. 


\section{Figure Captions}

Figure 1. Structures of GaP p-i-n devices (left) and $\operatorname{In}(\mathrm{N}) \mathrm{P}$ QD-embedded GaP p-i-n devices (right).

Figure 2. AFM images of InP QDs (left) and In(N)P QD (right). Both images are squares with sides $1 \mu \mathrm{m}$ in length.

Figure 3. PL spectra of InP QDs and InNP QDs at $4 \mathrm{~K}$ obtained using an excitation wavelength of $532 \mathrm{~nm}$ and a pumping energy density of $1 \mathrm{~W} \mathrm{~cm} \mathrm{~cm}^{-2}$. Inset: band diagram of the $\mathrm{In}(\mathrm{N}) \mathrm{P} / \mathrm{GaP}$ system. The solid black lines represent the $\Gamma$-derived valence bands. The dotted lines represent the X-derived band in GaP. The solid blue line represents the $\Gamma$ derived band without $\mathrm{N}$ and the green line represents the lower-energy band formed in InNP QDs after $\mathrm{N}$ incorporation. The solid red arrow indicates the $\Gamma-\Gamma$ transition while the dashed red arrow indicates the $\mathrm{X}-\Gamma$ transition.

Figure 4. Current-density versus voltage $(J-E)$ behavior of $\mathrm{GaP}$ (black), InP QDembedded (red) and InNP QD-embedded (blue) electrodes in contact with a $\mathrm{CH}_{3} \mathrm{CN}$ solution containing the $\mathrm{FeCp}_{2}{ }^{+/ 0}$ redox couple and $1.0 \mathrm{M} \mathrm{LiClO}_{4}$ as a supporting electrolyte. Illumination was provided by an ELH-type tungsten-halogen lamp calibrated to provide $100 \mathrm{~mW} \mathrm{~cm}^{-2}$ of simulated AM 1.5 illumination at the surface of the electrodes.

Figure 5. External quantum yield ( $\left.\Phi_{\text {ext }}\right)$ of GaP, InP QD-embedded GaP, and InNP QDembedded $\mathrm{GaP}$ samples in contact with a $\mathrm{CH}_{3} \mathrm{CN}$ solution containing the $\mathrm{FeCp}_{2}{ }^{+/ 0}$ redox couple and $1.0 \mathrm{M} \mathrm{LiClO}_{4}$ as a supporting electrolyte. 


\section{Vitae}

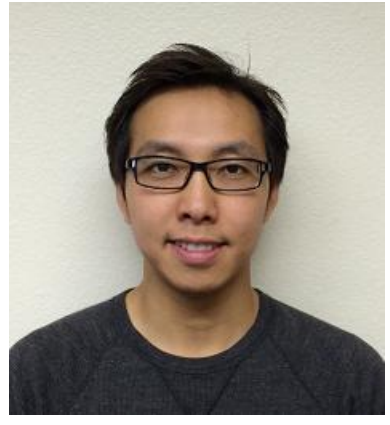

Yanjin Kuang received his Ph.D. degree in Physics at the University of California, San Diego (UCSD) in 2014 for his work on dilute nitride based intermediate band solar cell materials. Prior to his doctoral study, he obtained his M.S. degree and B.S. degree both in Physics at UCSD and at University of Science and Technology of China. His research focuses on molecular beam epitaxy and optical and electrical characterization of III-V semiconductor materials in thin film, nanowire and quantum dot structures.

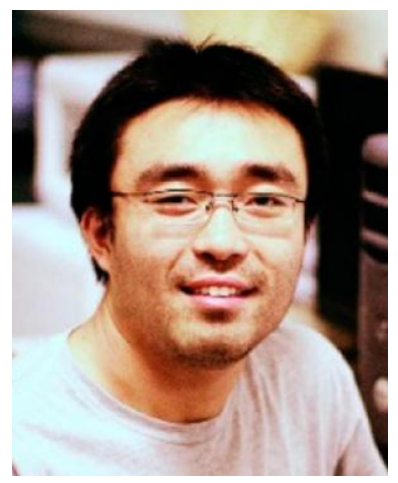

Ke Sun obtained his Ph.D. degree in Electrical and Computer Engineering from the University of California at San Diego in 2013 for his work on synthesis/characterization of nanomaterials and heterogeneous surface modification of $\mathrm{Si}$ for optoelectronics and solar water splitting. He is currently a postdoctoral researcher at the Joint Center of Artificial Photosynthesis (DOE Energy Innovation Hub) under the supervision of Professor Nathan Lewis at Caltech. He is continuously committed to solarfuel research. Specifically, he is focusing on development of protective coatings for unstable photoelectrodes in aqueous environment, optimization of electrocatalyst loading, and optimization of Si microwirebased photoelectrodes.

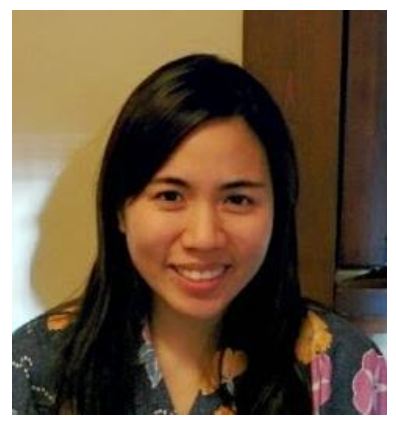

Supanee Sukrittanon is a Ph.D. candidate in the Materials Science and Engineering Program at the University of California, San Diego (UCSD). She also received her M.S. degree in the same program at UCSD. Prior to UCSD, she received her B.S. degree in Physics at Chulalongkorn University in Thailand. Her research focuses on III-V semiconductors, MBE growth, and optical devices.

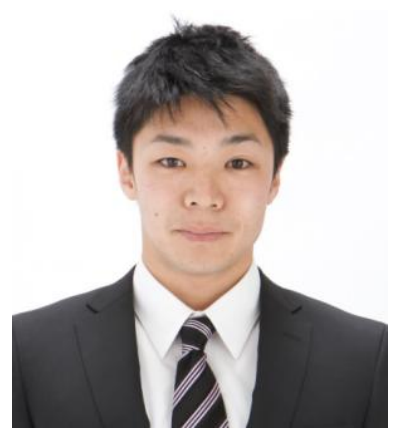

Ko Takabayashi received his bachelor's degree in materials engineering from Toyota Technological Institute (TTI), Japan in 2013. He is currently pursuing master's program at TTI under the direction of Professor Itaru Kamiya. From August to October in 2013 he was a visiting student in Professor Tu's group at University of California San Diego. His current research is focused on the growth of III-V semiconductor nanostructures and their $\mathrm{nm}$-scaled characterization with Kelvin probe force microscopy combined with atomic force microscopy. 


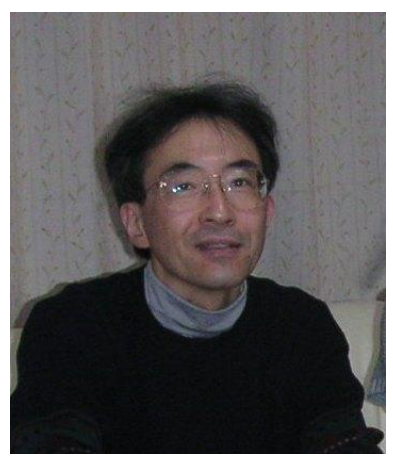

Itaru Kamiya received his BS, MS, and Ph.D. in Applied Physics from The University of Tokyo, Japan. He has been a Professor at Toyota Technological Institute, Nagoya, Japan, since 2004. Prior to taking his present position, he headed the Nanomaterials Lab at Mitsubishi Chemical Corp. after working at RIKEN, Bellcore, Imperial College, and The University of Tokyo. His research interest lies in the growth of semiconductor nanostructures using epitxial and chemical techniques, and the optical \& electronic properties of these materials particularly those related to surfaces and interfaces.

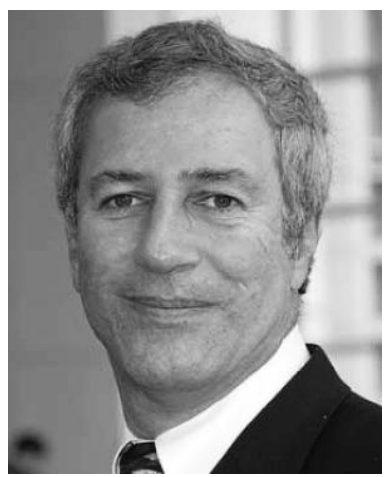

Dr. Nathan Lewis, George L. Argyros Professor of Chemistry, has been on the faculty at the California Institute of Technology since 1988 and has served as Professor since 1991. He has also served as the Principal Investigator of the Beckman Institute Molecular Materials Resource Center at Caltech since 1992, is the Principal Investigator of the Joint Center for Artificial Photosynthesis, the DOE's \$122 MM Energy Innovation Hub in Fuels from Sunlight. Dr. Lewis received his Ph.D. in Chemistry from the Massachusetts Institute of Technology. He has been an Alfred P. Sloan Fellow, a Camille and Henry Dreyfus TeacherScholar, and a Presidential Young Investigator.

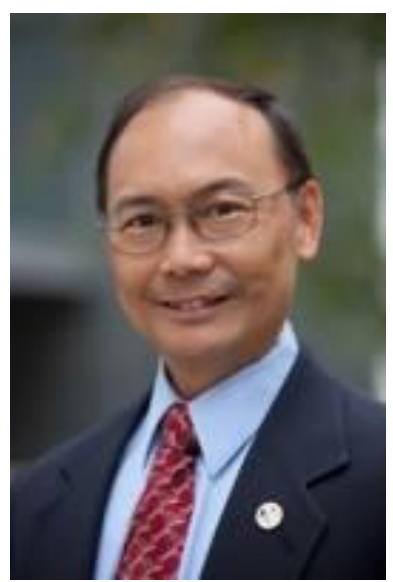

Charles $\mathrm{Tu}$ is a Distinguished Professor of electrical and computer engineering (ECE) at the UCSD Jacobs School of Engineering. He joined the UCSD faculty in 1988, and was appointed associate dean of the Jacobs School from 2004 to 2014, after serving from 1999 to 2003 as chair of the ECE department. Tu's research interests include novel III-V compound semiconductor heterostructures and nanostructures for electronic, optoelectronic and photovoltaic devices. He was a distinguished member of AT\&T Bell Laboratories technical staff from 1980 to 1988. He earned his Ph.D. in Engineering and Applied Science from Yale University in 1978 and his B.Sc. (Hon.) in Physics from McGill University in 1971. 